\title{
Appropriateness of Hospital Equipment Ergonomic Indices to Nurse's anthropometric Dimensions
}

\author{
Fatemeh Abareshi', Reza Hekmatshoar ${ }^{2}$, Sedighe Rastaghi ${ }^{3,4}$, Zahra Sharifi, ${ }^{5, *}$
}

${ }^{1}$ MSc in Occupational Health, Lecturer, Occupational Health Department, School of Public Health, Sabzevar University of Medical Sciences, Sabzevar, Iran

${ }^{2}$ PhD student in Environmental Pollution, Lecturer, Occupational Health Department, School of Public Health, Sabzevar University of Medical Sciences, Sabzevar, Iran

${ }^{3}$ PhD Student in Biostatistics, Department of Biostatistics, Sabzevar University of $\mid$ Medical Sciences, Sabzevar, Iran

${ }^{4}$ PhD Student in Biostatistics, Department of Biostatistics, Faculty of Health, Mashhad University of Medical Sciences, Mashhad, Iran

${ }^{5}$ MSc in Ergonomics, Occupational Health Department, School of Public Health, Social Determinants of Health Research Center, Sabzevar University of Medical Sciences, Sabzevar, Iran

* Corresponding Author: Zahra Sharifi, Occupational Health Department, School of Public Health, Social Determinants of Health Research Center, Sabzevar University of Medical Sciences, Sabzevar, Iran._Email: Sharifi_12_ohs@yahoo.com

\begin{tabular}{l} 
Received: $10 / 01 / 2019$ \\
Accepted: $16 / 03 / 2019$ \\
\hline How to Cite this Article: \\
Abareshi F, Hekmatshoar R, \\
Rastaghi S, Sharifi Z. Appropria- \\
teness of Hospital Equipment \\
Ergonomic Indices to Nurse's \\
anthropometric Dimensions. J \\
Occup Hyg Eng. 2019;5(4): $33-$ \\
40. DOI:10. 29252/johe.5.4.33
\end{tabular}

\section{Abstract}

Background and Objective: Lack of anthropometric data in order to design workstations and equipment can cause work-related injuries; therefore, it is necessary to create anthropometric database. The aim of the present study was to evaluate the appropriateness of hospital equipment ergonomic indices to female nurses' anthropometric dimensions in one of the teaching and therapeutic hospitals of Sabzevar, Iran.

Materials and Methods: In this cross-sectional study, 96 female nurses were selected using convenience sampling method. In the first phase, the participants completed the demographic questionnaire and body map checklist. Then, in addition to height and weight, 14 anthropometric dimensions of the subjects were measured by an expert in ergonomics using Calipers. Furthermore, in order to compare the participants' ergonomic dimensions with equipment dimensions in hospital, the dimensions of the ten most used equipment in the hospital were measured. The data were analyzed by SPSS software (version 17) using the Chi-square test, Mann-Whitney $U$ test, Fisher's exact test, and Student's t-test. P-value less than 0.05 was considered statistically significant.

Results: The findings of the present study indicated that high prevalence of musculoskeletal pain among female nurses, especially in the neck, waist, back, shoulder, and knee regions were reported as $73.7 \%, 70.62 \%, 69.38 \%$, $14 / 55 \%$, and $32 / 48 \%$, respectively. Moreover, the investigation of the participants' anthropometric dimensions and the measurement of equipment dimensions revealed that there was no appropriateness in many aspects, such as access limit, mid-shoulder height, standing elbow height, with the equipment, such as serum holder, drug trolley, medicinal shelf, hospital bead, and nursing station.

Conclusion: Despite the differences in the physical dimensions of female nurses, the equipment was not proportional to the participants, anthropometric dimensions in the present study. It is necessary to consider this issue in order to reduce the incidence of musculoskeletal disorders. 
dof: $10.29252 /$ johe. 5.4 .33

\title{
شاخصهاى ارتونومى تجميزات بيمارستانى با ابعاد آنترويومتريك
}

\section{براسى تناسب

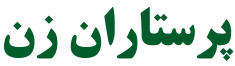

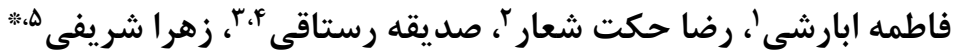

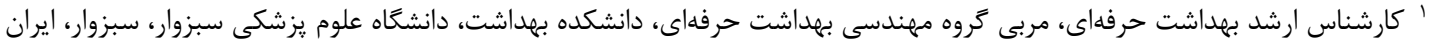

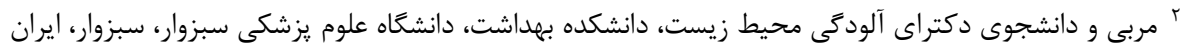

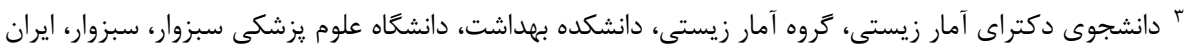

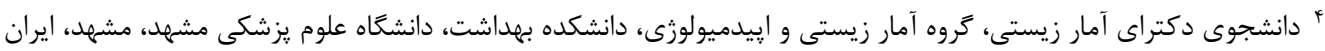

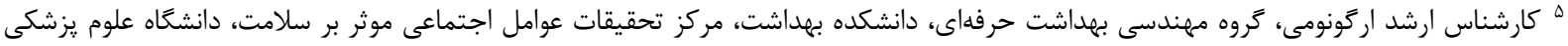
سبزوار، سبزوار، ايران

* نويسنده مسئول: زهرا شريفى، كروه مهندسى بهداشت حرفهاى، دانشكده بهداشت، مركز تحقيقات عوامل اجتماعى موثر بر سلامت، دانشكاه علوم يزشكى سبزوار، سبزوار، ايران. ايميل: Sharifi_12_ohs@yahoo.com

جكيده

سابقه و هدف: فقدان دادهاى آنترويومترى در طراحى ايستخاههاى كارى و تجهيزات مىتواند منجر به آنه

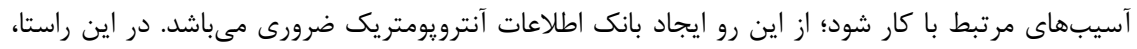

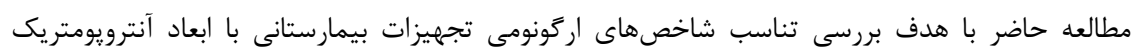

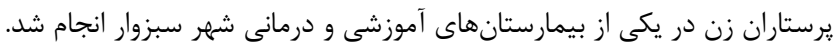

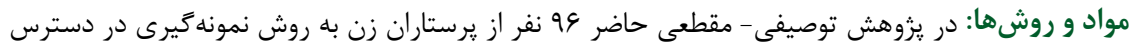

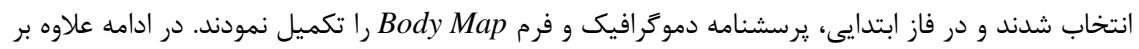

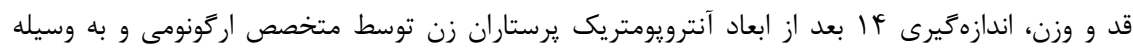

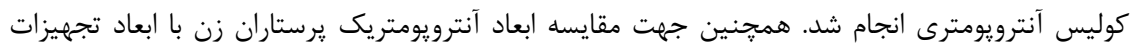

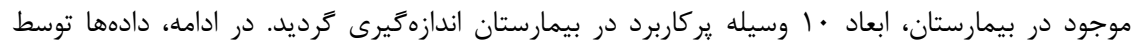

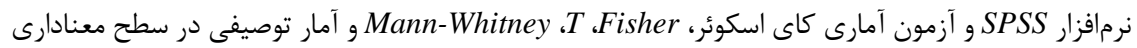

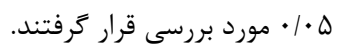

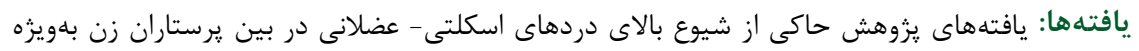

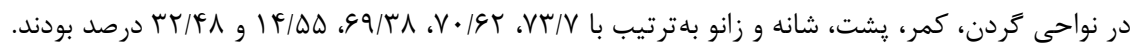

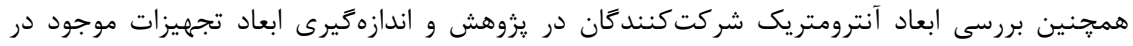

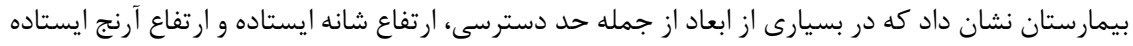

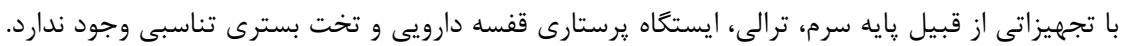

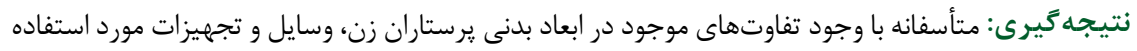

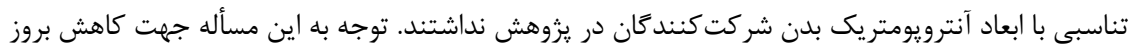
اختلالات اسكلتى - عضلانى امرى ضرورى مى باشد.

وارَّان كليدى: آنترويومترى؛ اركونومى؛ يرستاران؛ تجهيزات بيمارستانى

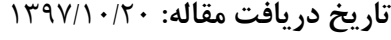

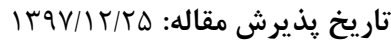

تمامى حقوق نشر براى دانشعاه علوم يزشكى همدان محفوظ است.

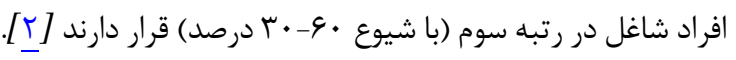

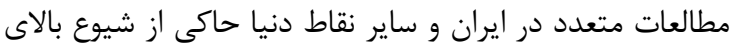

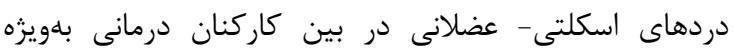

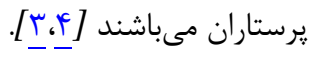

مقلمهه اختلالات اسكلتى - عضلانى مرتبط با كار يكى از مشكلات

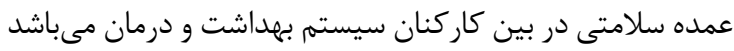

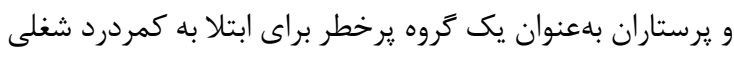

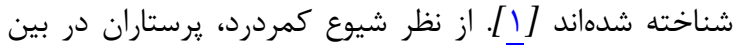


ويزگگىهاى جسمانى و روانى كاربران احساس مىشود. بر خلاف

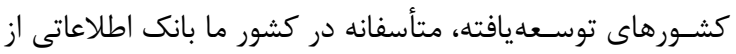

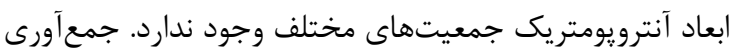

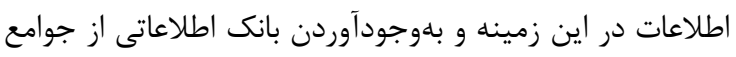

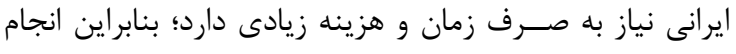

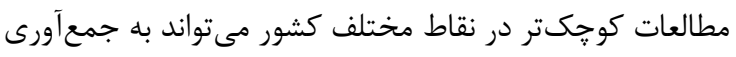

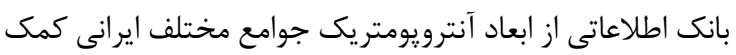

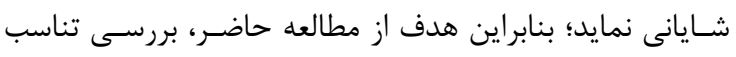

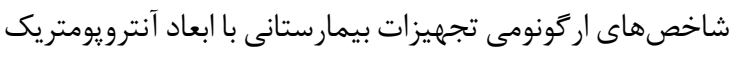

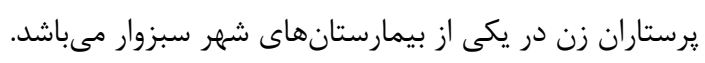

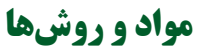

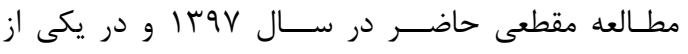

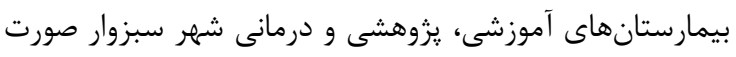

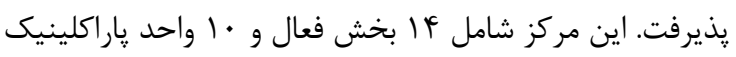
مىباشد.

\section{شركت كنندكان در يثروهش}

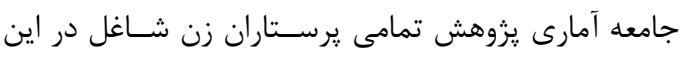

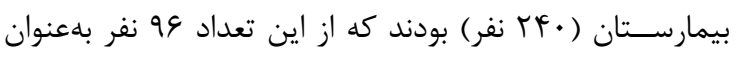

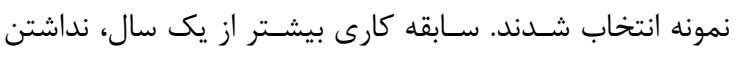

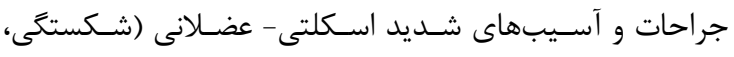

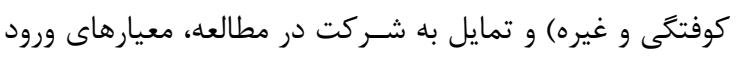

به مطالعه بودند.

حجم نمونه با استفاده ار نرمافزار

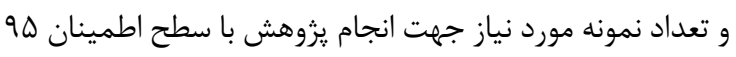

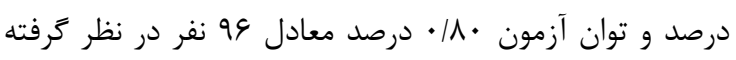

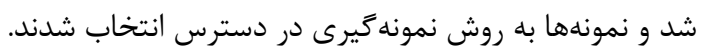

\section{ابزار جمعآورى /طلاعات}

يرسـشنامهاى مشتمل بر دو قسمت اطلاعات دمات دموكرافيك

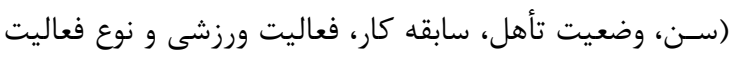

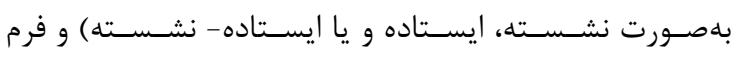
Body Map

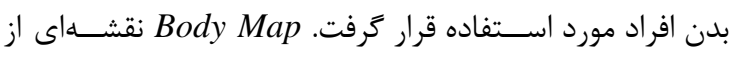

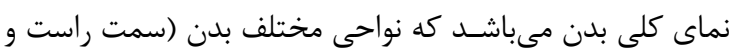

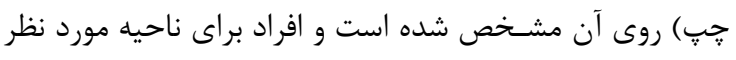

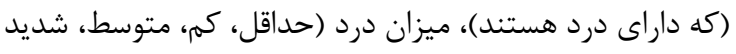

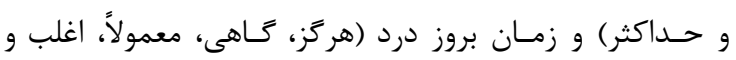
هميشه) را مشخص مئمايند.

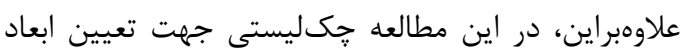

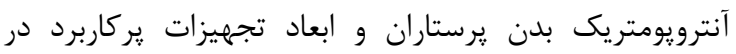

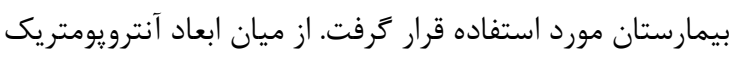

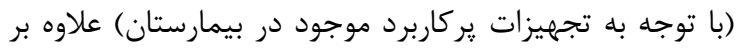

در اين ميان اكر محيط كارى و وسـايل و تجهيزات مورد

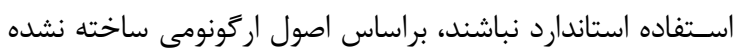

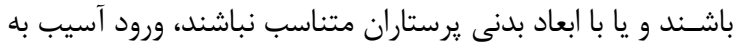

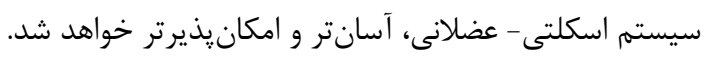

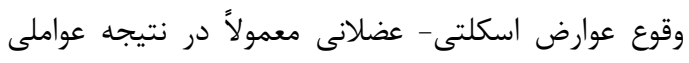

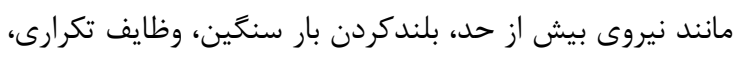

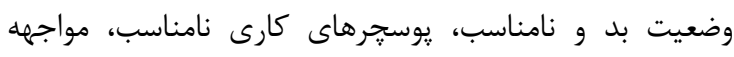

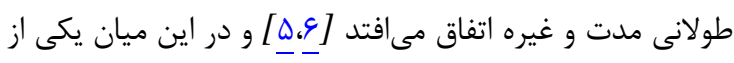

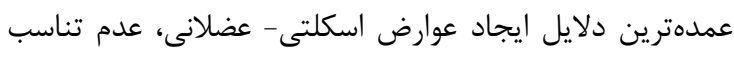

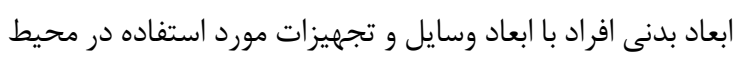

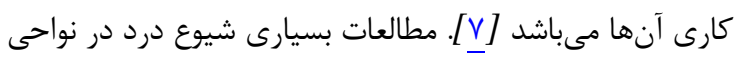

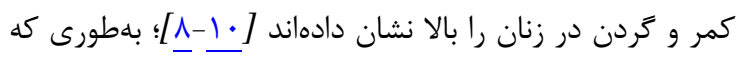

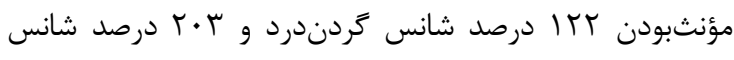

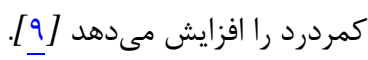

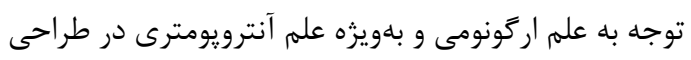

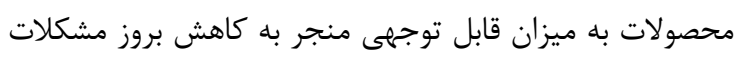

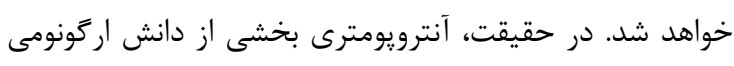

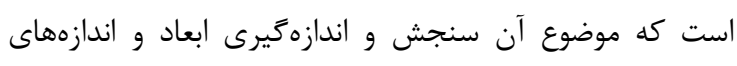

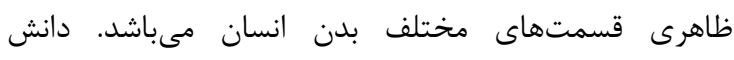

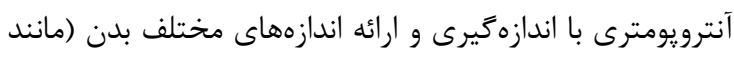

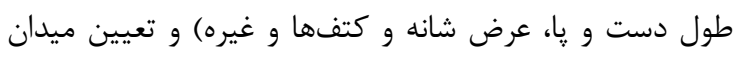

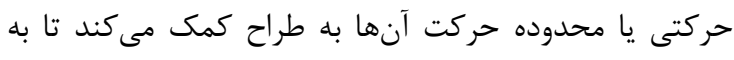

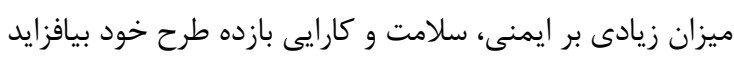

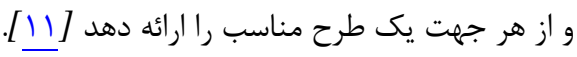

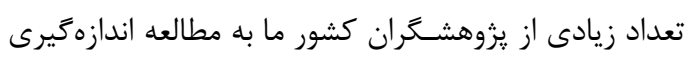

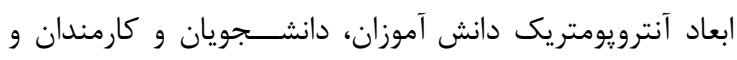

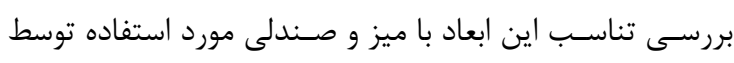

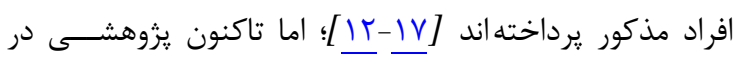

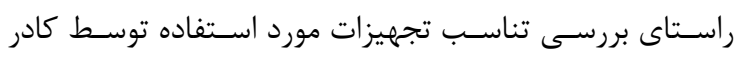

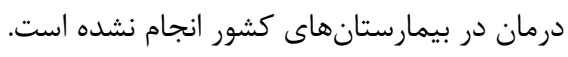

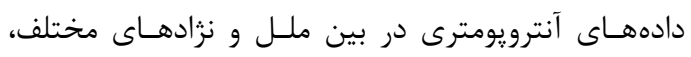

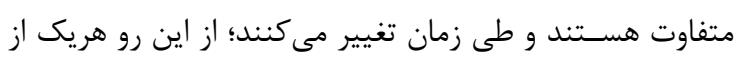

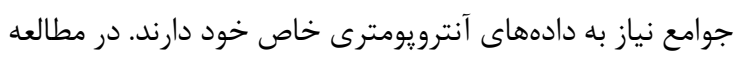

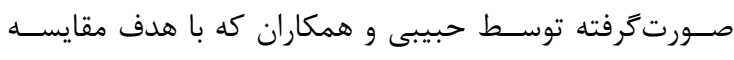

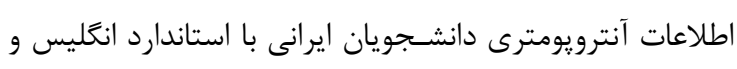

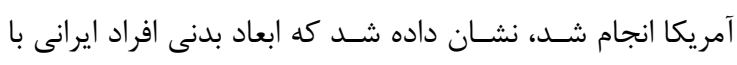

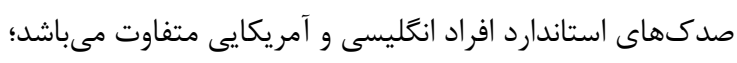

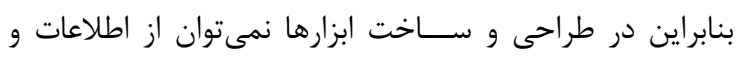

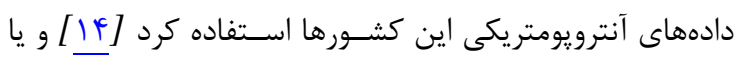

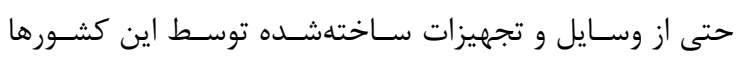
استفاده نمود. با توجه به نقش يرسـتار در جامعه امروز و اهميت تناسـب آنتب

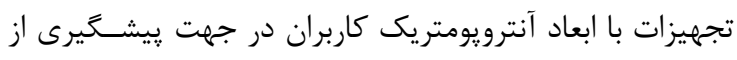

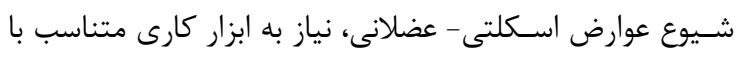


معنادارى (ه •|•>

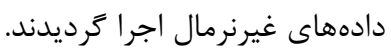

بافتهنا

ميانگين سنى شركت كنند

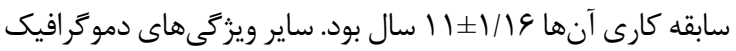
شركت كنند

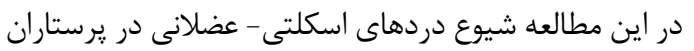

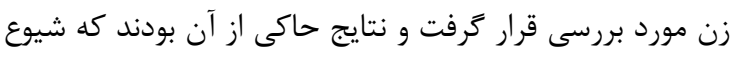

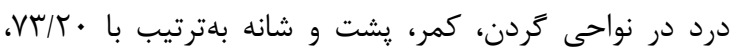

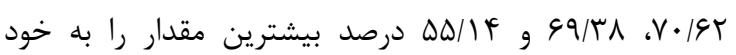

اختصاص دادهاند (نمودار ()).

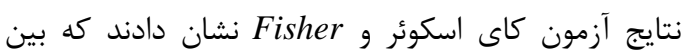

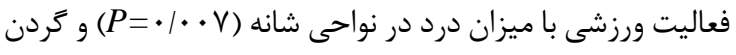

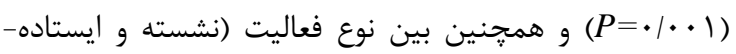

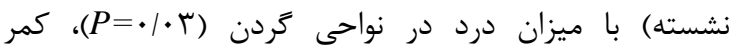

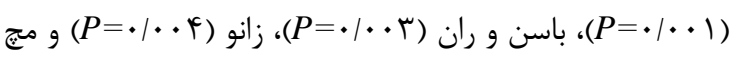

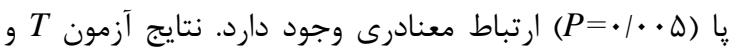
Mann-Whitney

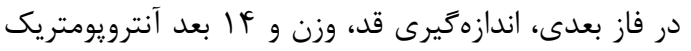

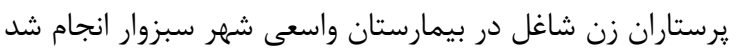

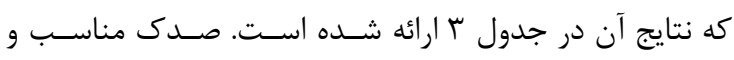

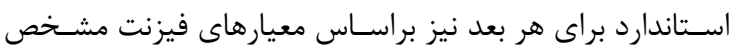

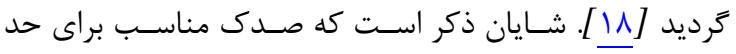

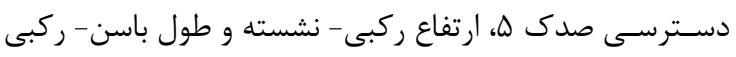

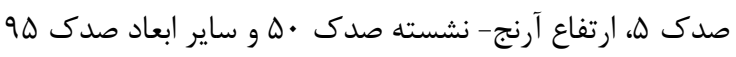
مىباشد. در ادامه، ابعاد تجهيزات مورد اسـتفاده در بيمارستان جهت جدول ا: ويزگ هاى دمو گرافيك يرستاران زن شركت كننده در مطالعه (N=94)

\begin{tabular}{|c|c|c|}
\hline تعداد (درصد) & & متغير \\
\hline$\Delta F(\Delta G / T \Delta)$ & متأهل & \multirow{2}{*}{ وضعيت تأهل } \\
\hline FT $(F T / V Q)$ & مجرد & \\
\hline$T G(Y V / \cdot \Lambda)$ & •r-r.r سال & \multirow{3}{*}{ سن } \\
\hline$F q(D) / \cdot F)$ & •ץ-ץ • • שال & \\
\hline$r \mid(T / / \Lambda \Lambda)$ & بيشتر از • F سال & \\
\hline$T r(T r / 9 T)$ & ا تا ه سال & \multirow{4}{*}{ سابقه كارى } \\
\hline$V F(V V / \cdot \Lambda)$ & بيشتر از ه سال & \\
\hline$V V(\Lambda \cdot / r \cdot)$ & كارشناسى & \\
\hline $19(19 / 1 \cdot)$ & كارشناسى ارشد & \\
\hline (rT & بله & \multirow{2}{*}{ فعاليت ورزشى } \\
\hline$G F(\& G \mid G V)$ & خير & \\
\hline $19(19 / 19)$ & ايستاده & \multirow{3}{*}{ نوع فعاليت } \\
\hline $\mid f(\mid F / \Delta \Lambda)$ & نشسته & \\
\hline$q \Gamma(\varepsilon \Delta / \varepsilon r)$ & ايستاده- نشسته & \\
\hline
\end{tabular}

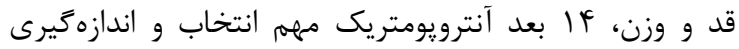

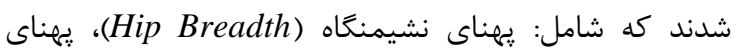
عرضى آرنجها (Elbow - to -Elbow Breadth)، ارتفاع شانه-

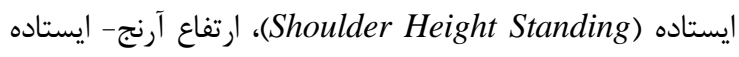
(Elbow Height Standing)، ارتفاع نشسته (Sitting Height)، ارتفاع آرنج- نشسته (Elbow Rest Height Sitting)، ارتفاع (Shoulder)،

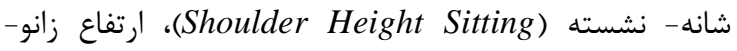
نشسته (Knee Height Sitting)، ارتفاع ركبى- نشسته (Sitting) (Popliteal Height Sitting) (Buttock- Knee Length Sitting) (Buttock- Popliteal Length)، طول شانه آرنج- نشسته باسن ركئ، - Shoulder - Elbow Length Sitting)

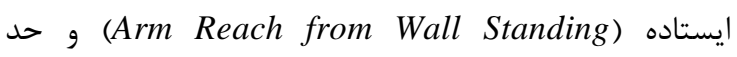
دسترسى عمودى - ايستاده (Vertical Grip Reach) بودند.

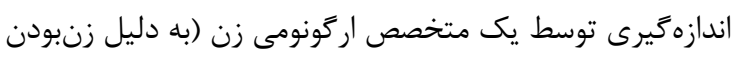

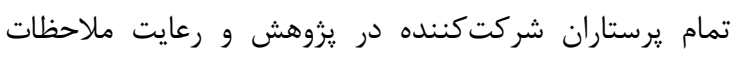

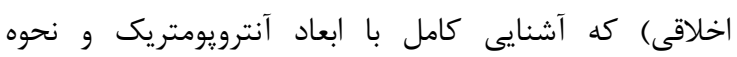

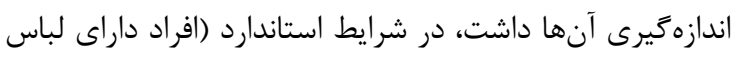

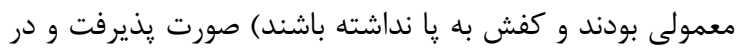

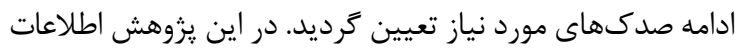
جمع آورىشده از ابعاد بدن از نوع اطلاعات استاتيكى بودند و كليه

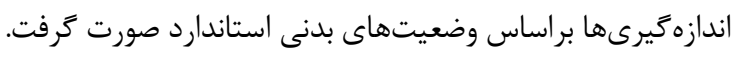

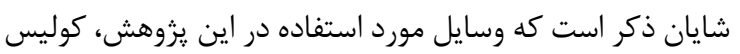

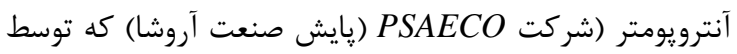

شركت سازنده كاليبره شده است) و ترازو بودند (شكل (1).

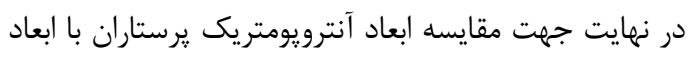

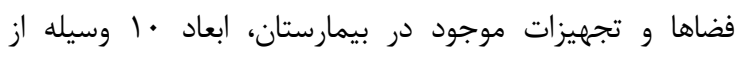

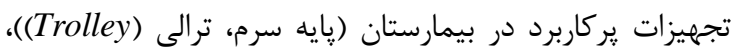

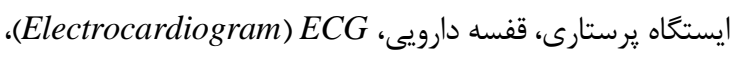

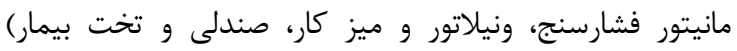
اندازمكيرى كرديد.

SPSS 17 تجزيه و تحليل دادهها نيز با استفاده از نرمافيل

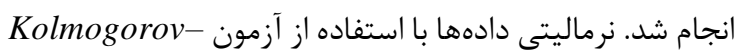
بررسى كرديد و آزمون آمارى كاى اسكوئر، Mann-Whitney T T Fisher

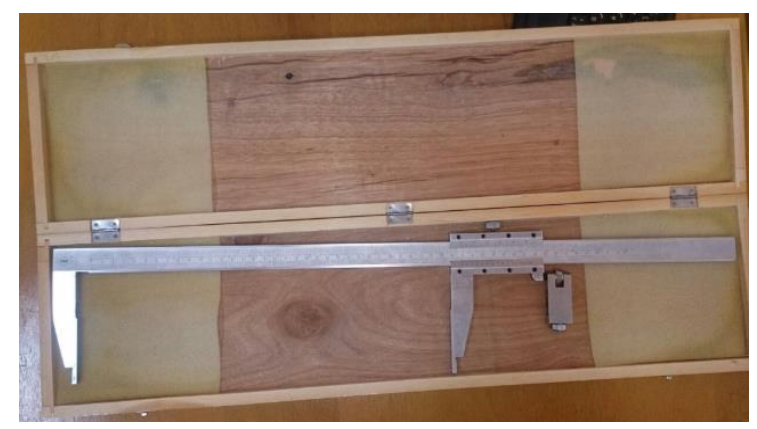

شكل ا: كوليس آنترويومتر 
- Series1

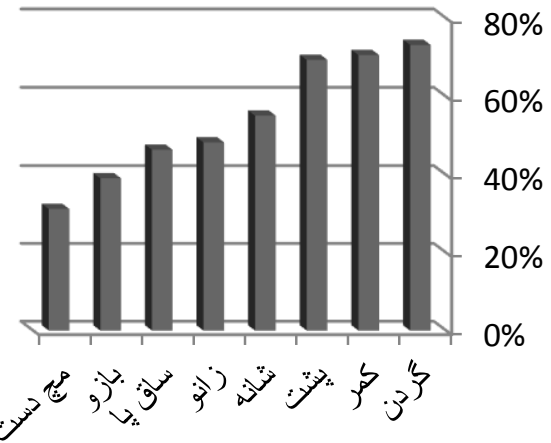

نمودار ا: شيوع دردهاى اسكلتى - عضلانى در يرستاران زن شركتكننده در مطالعه

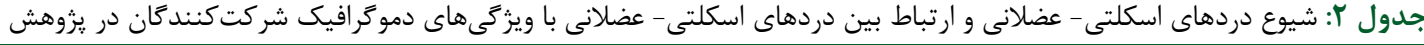

\begin{tabular}{|c|c|c|c|c|c|c|}
\hline نوع فعاليت & فعاليت ورزشى & سابقه كارى & وضعيت تأهل & سن & سطح معنادارى & اندامهاى بدن \\
\hline$\cdot / \cdot$ ץ $^{*}$ & •/・r萧 & $\cdot \mid F \Delta I$ &.$/ \cdot 14$ & $\cdot / \cdot r^{*}$ & سطح معنادارى & كردن \\
\hline$\cdot /\left.\cdot\right|^{\text {絭奠 }}$ & ( & 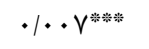 & $\cdot / \cdot v$ & $\cdot / T / \Delta$ & سطح معنادارى & كمر \\
\hline$\cdot|f| x \mid$ & $\cdot \mid \cdot v 1$ & • &.$/ 4 I t$ & $.1 .9 T$ & سطح معنادارى & ل \\
\hline$\cdot / \cdot V \Delta$ & $\cdot \cdot \cdot r^{\text {w:*n: }}$ & س & $\cdot 11 \cdot r$ & $\cdot 1 \cdot \cdot 1 *$ & سطح معنادارى & شانه \\
\hline 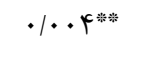 & $\cdot / \wedge \Delta$. & 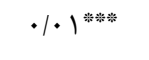 & $\cdot 19 \cdot 1$ & $\cdot / \cdot c^{c *}$ & سطح معنادارى & زانو \\
\hline. & $\cdot \mid \Lambda \Delta F$ &.$/ 1 T \Delta$ & $\cdot 1 \cdot V T$ & . /QRT & سطح معنادارى & ساق یا \\
\hline$\cdot 11 \cdot 4$ & - / K49 &.$/ 1 T r$ & - TER & $\cdot / T V T$ & سطح معنادارى & بازو \\
\hline$\cdot|\Delta \Delta|$ & ( & $\cdot / \cdot v 1$ & - MFT & - IVAT & سطح معنادارى & مج دست \\
\hline - /Tra &.$/ 1 K r$ & .1 .91 &.$/ 1 Y F$ & .1 .90 & سطح معنادارى & ساعد و آرنج \\
\hline$\cdot \mid \cdot r r^{* * * *}$ & - IA $4 \Delta$ & $\cdot|\Delta| F$ & $\cdot 1 \cdot V F$ & $\cdot / \cdot \wedge \vee$ & سطح معنادارى & باسن و ران \\
\hline$\cdot 1 \cdot \cdot \Delta^{\text {***as}}$ & .1 .90 & $.|| \Delta \mid$ & 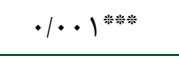 & $\cdot / \cdot r^{*}$ & سطح معنادارى & مج يا \\
\hline
\end{tabular}

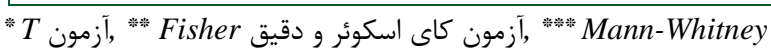

جدول "ז: ابعاد آنترويومتريك شركت كنند

\begin{tabular}{|c|c|c|c|c|c|c|c|}
\hline صدى نود و & صنجاهم & صدجى & ميانغين土|نحراف & حداكثر & حداقل & صداى هر مناسب & ابعاد \\
\hline IVT & 19. & $\mid \Delta F / \Lambda$. & $191 / 9 \cdot \pm \Gamma / 9 V$ & IVA & IQT & صدى هو & قد" \\
\hline 91 & GT & $F N / \Lambda$. & $G F / I r \pm r / g T$ & $9 F$ & iv & صدى DQ & وزن (كيلوكرم) \\
\hline$r \Delta / 1 \Lambda$ & $r r / \Delta \Lambda$ & 19 & $r F / F \cdot \pm r / q \Delta$ & rQ/AT & IN/Ar & صدى هو & 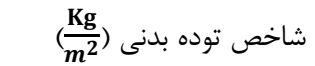 \\
\hline$|F| / F$. & $r \varepsilon / \uparrow$. & $r T / \Lambda$. & $r \psi / \backslash \Lambda \pm \psi / \cdot 1$ & $F F / Q$. & $r r / Q \cdot$ & صدى هి & يهناى نشيمنگًاه \\
\hline$\Delta \varphi$ & Ft & rr/A. & $F / / \Delta) \pm F / \Lambda q$ & $\Delta 1$ & $r r / \Delta$. & صدى هף & بهناى عرضى آرنجها \\
\hline $\mid F V / F$. & IrF & ITF & $\mid r Y / D S \pm T / Q T$ & IFA & $I T F$ & صدى هף & ارتفاع شانه ايستاده \\
\hline $1 \cdot \Delta / \Delta \cdot$ & $q \wedge / \Delta$. & $\wedge 9 / \Delta$. & QV/TTEI/IT & $|r| / V$. & $\wedge \vee$ & صدى ه & ارتفاع آرنج ايستاده \\
\hline$\wedge 9 / 9$. & vq & $99 / 9$. & $\vee q / r q \pm r / r r$ & 94 & $\varepsilon V / \Delta$. & صدى هو & ارتفاع نشسته \\
\hline 41 & $\Delta \cdot$ & $r \cdot 19$. & $r q / V \cdot \pm r / \wedge \Delta$ & gf & mF & صدى 99 & ارتفاع شانه نشسته \\
\hline$T Y / T$. & Tr & 19 & $r \mid / F T \pm 1 / 9 \varphi$ & $T F / Q$. & 19 & صدى •ט & ارتفاع آرنج- نشسته \\
\hline$\Delta \cdot / \kappa$ & FT & rN/T. & $\leftarrow \Psi / Y \Lambda \pm r / \varepsilon \Delta$ & QI & rv & صدى هף & ارتفاع زانو نشسته \\
\hline$\varphi \notin / \Gamma$. & $r \Delta / \Delta$. & $r \cdot / \Lambda$. & $r \varepsilon / 0 \cdot \pm r / r \varepsilon$ & $4 q$ & $r \cdot 10 \cdot$ & صدك ه & ارتفاع ركبى نشسته \\
\hline $41 / T$. & FV & f. & $F V / V F \pm T / r)$ & Va & f. & صدى هף & طول زانو- نشيمنحاه \\
\hline$\kappa \mu / \Lambda$. & rV & $r M / r$. & $r V / \backslash \Delta \pm 1 / \cdot r$ & $\uparrow q / b$. & $r$. & صدى ه & طول باسن - ركبى \\
\hline Fr/g. & $r F / Q$. & $r \Lambda / F$. & $r F / V F \pm F / / Q$ & $F F / Q$ & זr & صدى ه9 & طول شانه- آرنج نشسته \\
\hline$V r / F$. & GK/D. & $\Delta V / T$ & $G r / F T \pm Y / I r$ & VF & $\Delta \varphi$ & صدى ه & طول بازو از ديوار ايستاده \\
\hline$r \mid r / F$. & 194 & $I V \Lambda / F$. & $19 / 11 \cdot \pm \% / 11$ & rID & $I V \Delta / T$. & صدى ه & حد دسترسى عمودى ايستاده \\
\hline
\end{tabular}


جدول f: ابعاد تجهيزات مورد استفاده توسط يرستاران زن شركت كننده در مطالعه

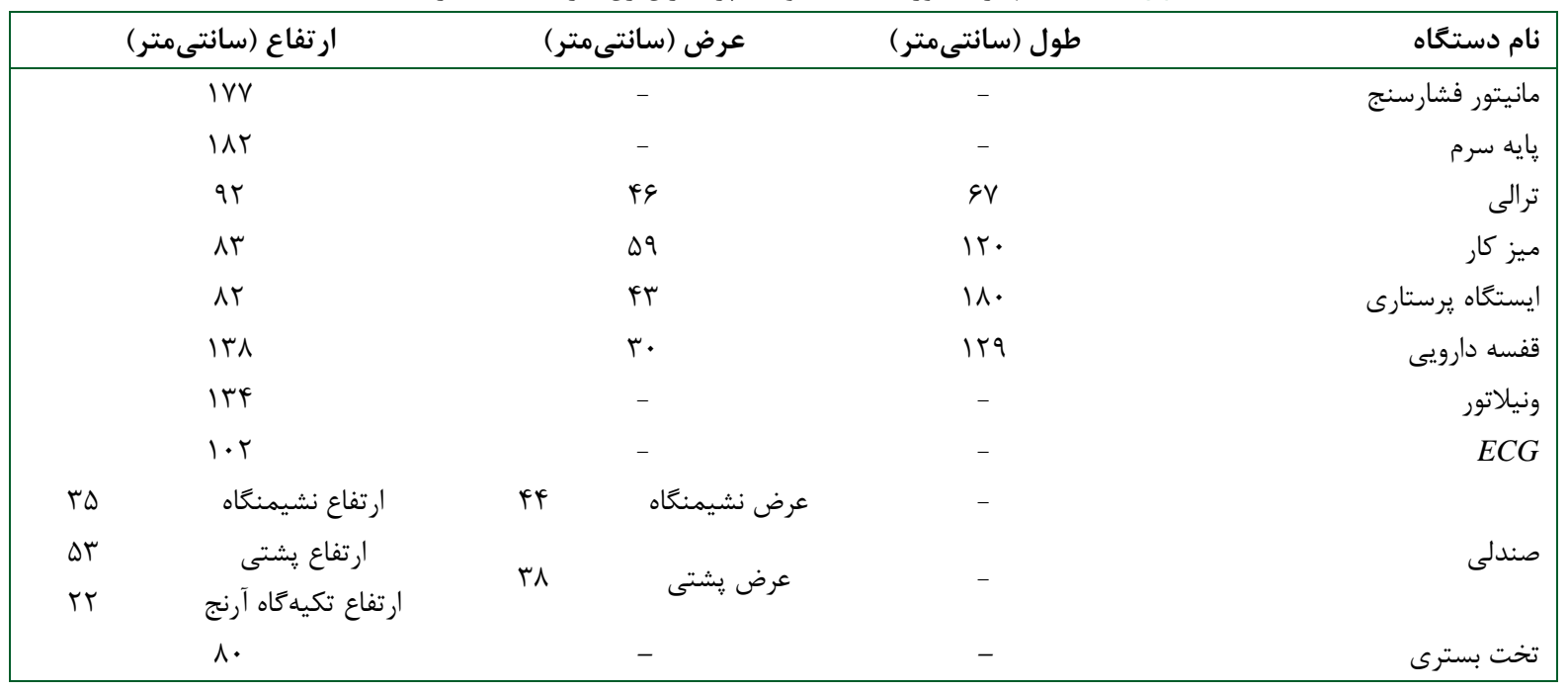

زانودرد و كمردرد ارتباط معنادارى داشت؛ بلطورى كه انجام

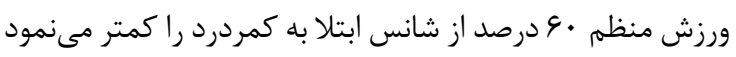

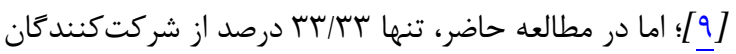

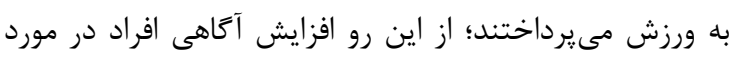
تأثيرات ورزش بر كاهش شيوع دردهاى اسكلتى - عضلانى، وجود برنامه ورزشى منظم در بين كاركنان بيمارستانى و نظارت

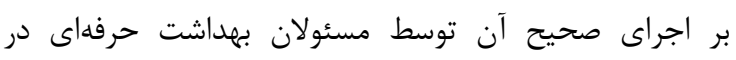
بيمارستانها توصيه مى

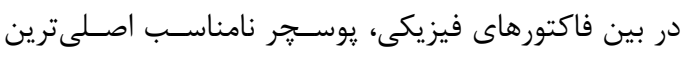

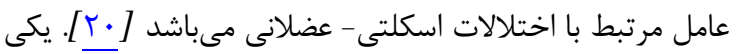

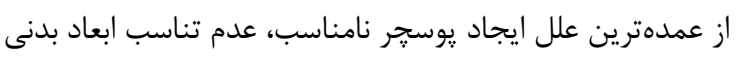

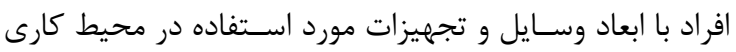

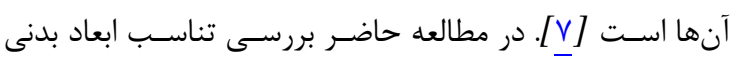

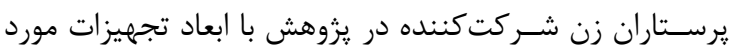

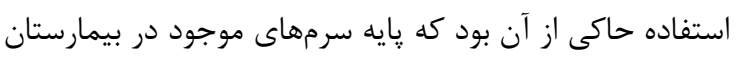

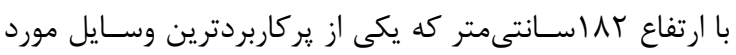

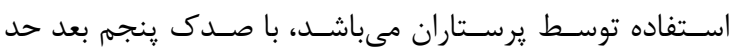

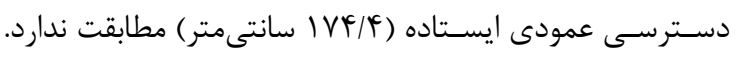

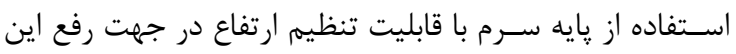

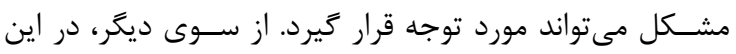

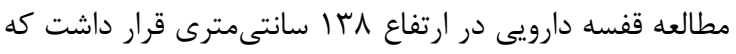

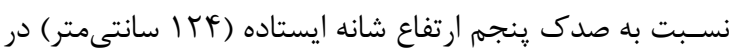

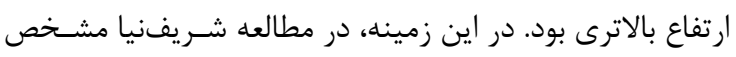

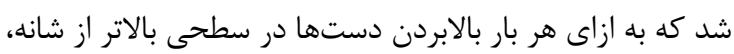

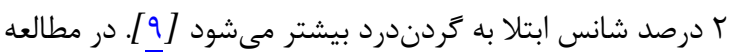

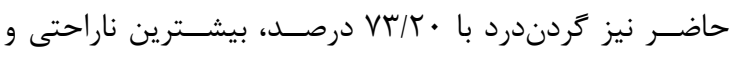

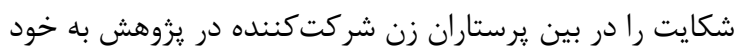

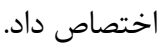

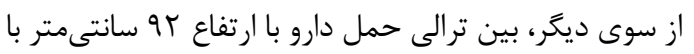

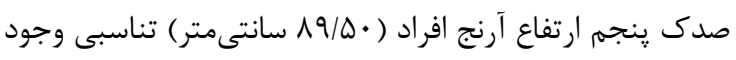

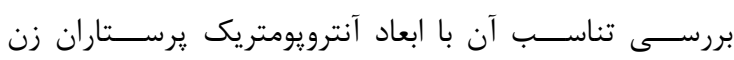

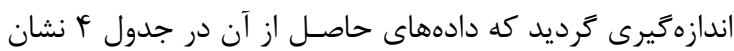

$$
\text { داده شده است. }
$$

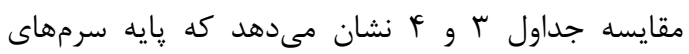

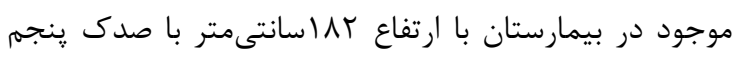

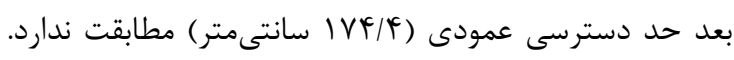

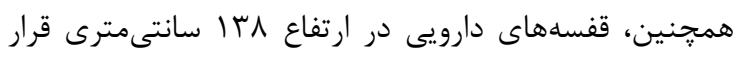

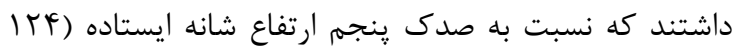

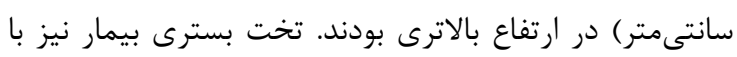

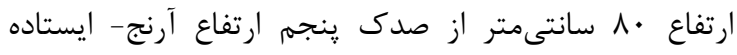

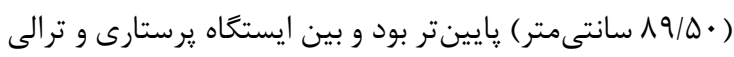

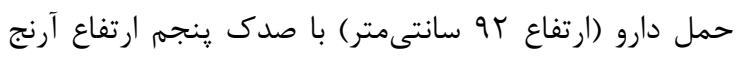
افراد تناسبى وجود نداشت. صندلىهاى مورد استفاده نيز مناسب نبودند.

هدف از يزوهش حاضر، بررسى ميزان شيوع دردهاى اسكلتى - ن.

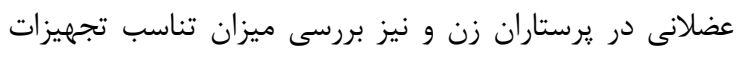

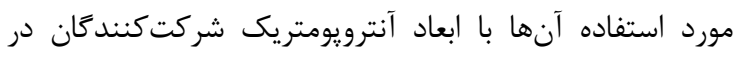

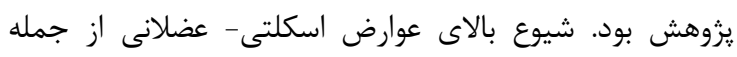

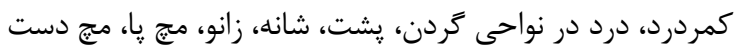

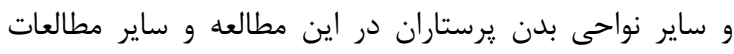

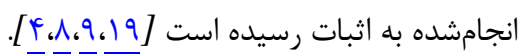

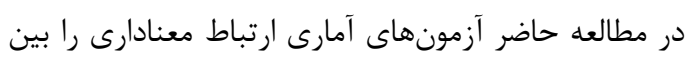

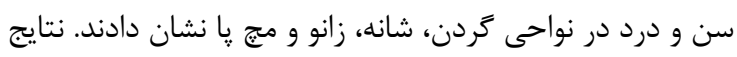

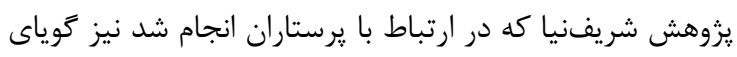

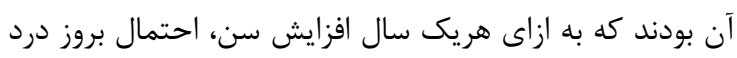

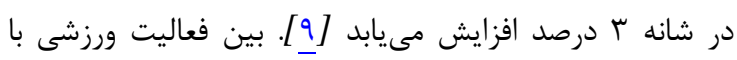
ميزان درد در نواحى شانه و گردن نيز رابطه معنادارى مشاهد دانده

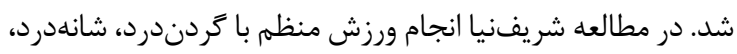


براســاس اين بانكى اطلاعاتى بلهـــت مورد نياز مىباشــد. در

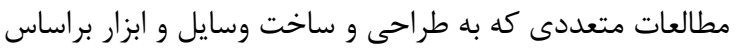

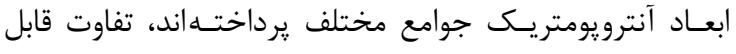

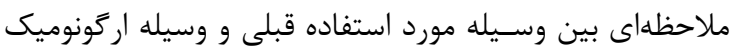

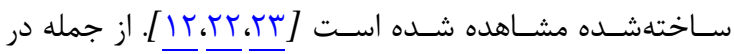

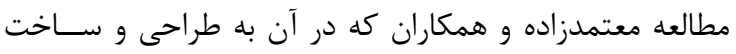

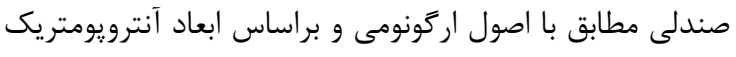

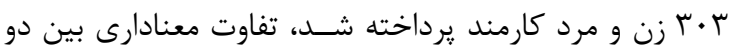

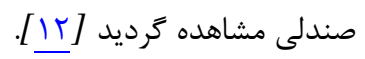

\section{نتيجه كيرى}

با توجه به اينكه ابعاد اغلب تجهيزات موجود در بيمارسـان

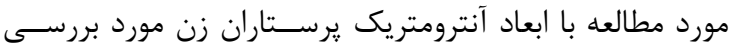

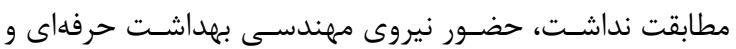

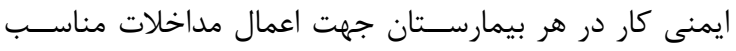

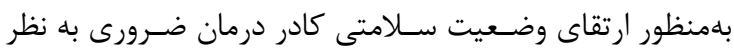

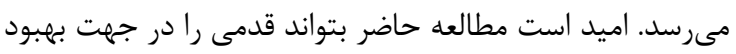
وضعيت اركونومى در بيمارستانها و كادر درمانى بردارد.

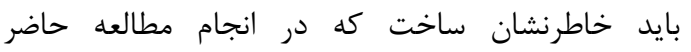
محدوديتهايى وجود داشت؛ از جمله اينكه تمامى شركت كنند

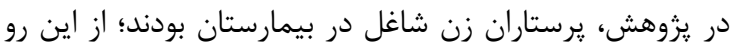

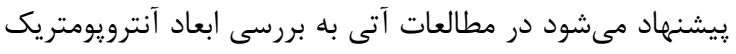

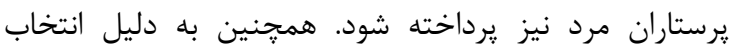
تجهيزات مشترك در بخشهاى مختلف بيمارستان، تعدادى از

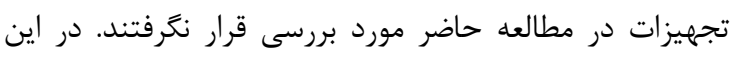

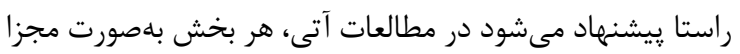

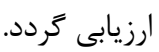

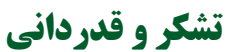

يزوهش حاضر حاصل طرح تحقيقاتى با شماره

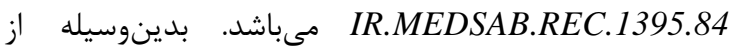

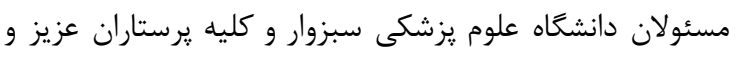
زحمت كش شركت كننده در اين يزوهش قدردانى مئى

\section{REFERENCES}

1. Ando $S$, Ono $Y$, Shimaoka $M$, Hiruta $S$, Hattori $Y$, Hori $F$, et al. Associations of self estimated workloads with musculoskeletal symptoms among hospital nurses. Occup Environ Med. 2000;57(3):211. DOI: 10.1136/oem.57.3.211

2. Mohseni Bandpei MA, Fakhri M, Ahmad Shirvani M, Bagheri Nesami M, Khalilian AR. Epidemiological aspects of low back pain in nurses. J Babol Univ Med Sci. 2005;26(7):40-3. [Persian]

3. Barzideh M, Choobineh AR, Tabatabaee HR. Job stress dimensions and their relationship to musculoskeletal disorders in Iranian nurses. Work. 2014;47(4):423-9. DOI: 10.3233/WOR-121585

4. Menzel NN. Psychosocial factors in musculoskeletal disorders. Crit Care Nurs Clin North Am. 2007;19(2):14553. PMID: 17512470 DOI: 10.1016/j.ccell.2007.02.006
نداشت. تختهاى بسترى بيمار (با ارتفاع •^ سانتىمتر) نيز از

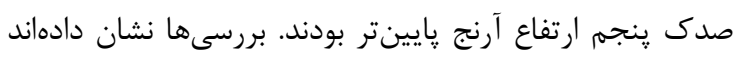

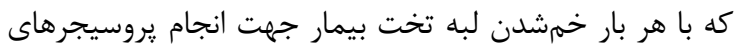

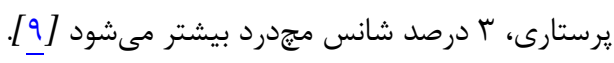

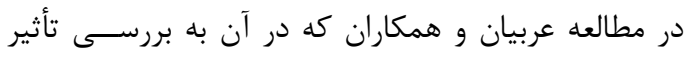

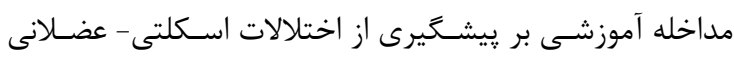

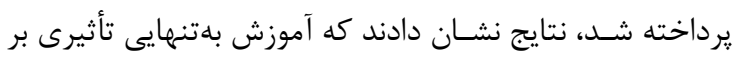

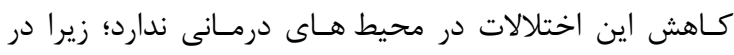

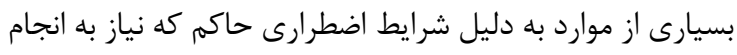

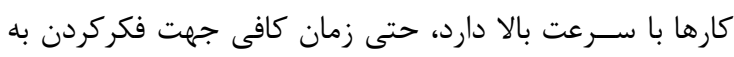

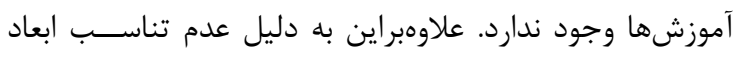

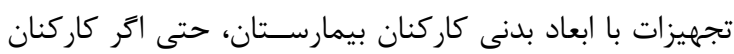

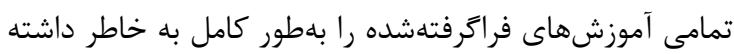

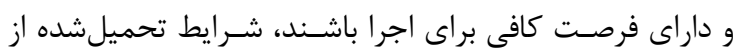

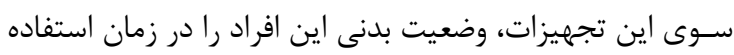

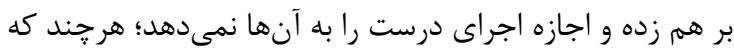

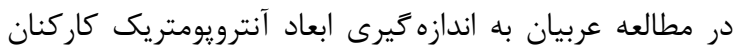

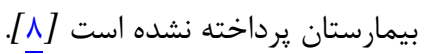

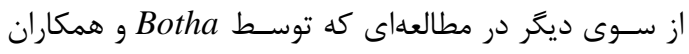

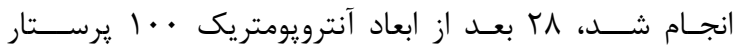

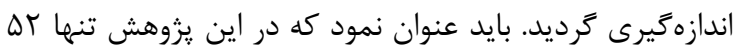

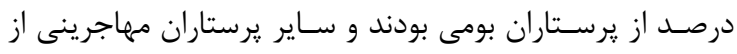

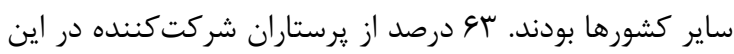

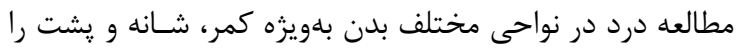

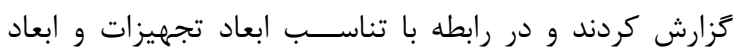

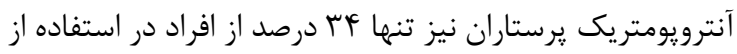

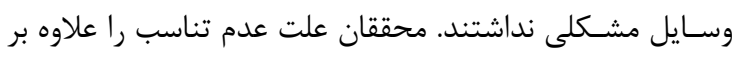

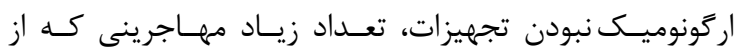

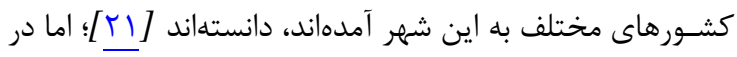

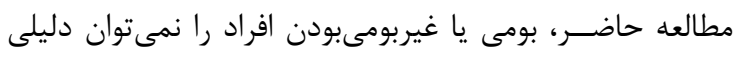

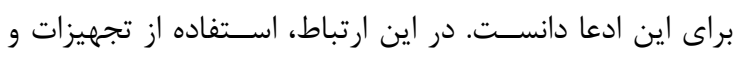

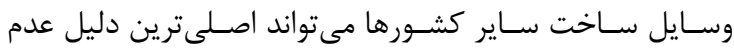

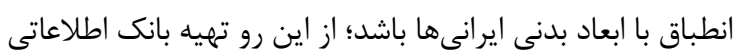

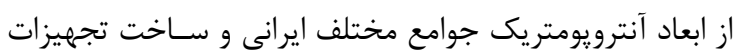

5. Sung HC, Pan YT. Ergonomic practices for packing tasks in a printing ink manufacturing factory. In: Lin DY, Chen $\mathrm{HC}$, editors. Ergonomics for all. London: CRC Press/Balkema; 2011.

6. Choobineh A, Tabatabaee S, Behzadi M. Musculoskeletal problems among workers of an iranian sugar-producing factory. Int J Occup Saf Ergon. 2009;15(4):419-24. DOI: 10.1080/10803548.2009.11076820

7. Juul-Kristensen B, Jensen C. Self-reported workplace related ergonomic conditions as prognostic factors for musculoskeletal symptoms: the "BIT" follow up study on office workers. Occup Environ Med. 2005;62(3):188-94. DOI: 10.1136/oem.2004.013920

8. Ali Arabian F, Motamedzade M, Golmohammadi R, Moghim Beigi A, Pir Hayati F. The impact of ergonomics intervention 
on musculoskeletal disorders among Nahavand Alimoradian hospital staff. J Ergon. 2013;1(1):23-32. [Persian]

9. Sharifnia SH, Haghdoost AA, Hajihosseini F, Hojjati $H$, Haghdoost AA. Relationship between the musculoskeletal disorders with the ergonomic factors in nurses. Koomesh. 2011;12(4):372-8. [Persian]

10. Lorusso A, Bruno $S$, L'abbate N. A review of low back pain and musculoskeletal disorders among Italian nursing personnel. Ind Health. 2007;45(5):637-44. PMID: 18057806

11. Vink P. Comfort and design: principles and good practice. New York: Library of Congress; 2005.

12. Motamedzade M, Hassan Beigi MR, Mahjoob H. Design and development of an ergonomic chair for Iranian office workers. J Zanjan Univ Med Sci. 2009;17(68):45-52. [Persian]

13. Heidarimoghadam $R$, Motamedzade $M$, Roshanaei $G$, Ahmadi $R$. Investigating the match between male primary students' anthropometric dimensions and existing furniture dimensions in Hamadan schools in 2013. J Ergon. 2014;2(1):9-18.

14. Habibi E, Sadeghi N, Mansouri F, Sadeghi MR, Ranjbar M. Comparison of Iranian student's anthropometric information and American and English standards. J Jahrom Univ Med Sci. 2012;10(2):22-30.

15. Heidarimoghadam $R$, Golmohammadi R, Roshanaei $G$, Zare $R$. Assessing the match between female primary students' anthropometric dimensions and furniture dimensions in Hamadan schools in 2013. Health Saf Work. 2015;5(1):47-
56. [Persian]

16. Varmazyar S. Design of school furniture based on anthropometric dimension girl students in Qazvine, Iran. Qom Med Sci Univ. 2008;3(2):40-5. [Persian]

17. Falahati M, Zokaei M, Sadeghi Naeini H, Moradi GR. Determination of variables and anthropometric indicators for classroom chair design (Study group: Tehran University medicine science-2011). Iran Occup Health. 2013;10(2):99108. [Persian]

18. Pheasant $S$. Bodyspace: anthropometry, ergonomics and the design of work: anthropometry, ergonomics and the design of work. Florida: CRC Press; 2014

19. Smith DR, Wei N, Kang L, Wang RS. Musculoskeletal disorders among professional nurses in mainland China. J Prof Nurs. 2004;20(6):390-5. PMID: 15599873

20. Choobineh A, Rajaeefard A, Neghab M. Perceived demands and musculoskeletal disorders among hospital nurses. Hakim Res J. 2007;10(2):70-5. [Persian]

21. Botha WE, Bridger RS. Anthropometric variability, equipment usability and musculoskeletal pain in a group of nurses in the Western Cape. Appl Ergon. 1998;29(6):481-90. DOI: 10.1016/S0003-6870(97)00081-1

22. Laios L, Giannatsis J. Ergonomic evaluation and redesign of children bicycles based on anthropometric data. Appl Ergon. 2010;41(3):428-35. DOI: 10.1016/j.apergo.2009.09.006

23. Mououdi MA. The determination of static anthropometry characteristics for designing and evaluating the comfort of saddle chair. Iran Occup Health. 2013;9(4):24-9. 